KUNS-1653

hep-ph/0003231

\title{
Novel Relations between Lepton and Quark Mixings
}

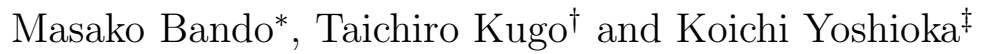 \\ * Aichi University, Aichi 470-0296, Japan \\ †, Department of Physics, Kyoto University Kyoto 606-8502, Japan
}

\begin{abstract}
A novel relation is found which gives the 2-3 lepton mixing angle $\theta_{\mu \tau}$ in terms of quark masses and CKM mixing:

$$
\tan \theta_{\mu \tau}=\left(m_{b} / m_{s}\right) V_{c b}
$$

This relation, which is remarkably in good agreement with the quark data and the large lepton mixing recently observed, is a kind of $S O(10)$ GUT relation similar to the celebrated bottom-tau mass ratio in $S U(5)$. The GUT models in which this relation hold should have a 'twisted $S O(10)$ ' structure between the second and third generations, in order to really explain the large lepton mixing, or equivalently, the right-hand side of the relation.
\end{abstract}

\footnotetext{
${ }^{*}$ E-mail address: bando@aichi-u.ac.jp

${ }^{\dagger}$ E-mail address: kugo@gauge.scphys.kyoto-u.ac.jp

${ }_{\ddagger}^{\ddagger}$ E-mail address: yoshioka@gauge.scphys.kyoto-u.ac.jp
} 
One of the most remarkable facts in recent particle physics is the very large lepton mixing observed in SuperKamiokande [1], which has revealed a sharp contrast to the quark sector where the CKM mixings are all small. Why is such a difference possible between the quark and lepton sectors? This is really a challenge to the attempts for grand unified theories (GUTs). Clearly any GUTs which treat the three generations of quarks and leptons as a mere repetition no longer work. We need some structural changes between the generations and a number of models accounting for the large lepton mixing angles have recently been proposed [2]

In our recent study of a supersymmetric $E_{6}$ grand unified model [3], we found a novel relation

$$
\tan \theta_{\mu \tau}=\frac{m_{b}}{m_{s}} V_{c b}
$$

or equivalently,

$$
\sin ^{2} 2 \theta_{\mu \tau}=\frac{4 V_{c b}^{2}\left(\frac{m_{s}}{m_{b}}\right)^{2}}{\left[V_{c b}^{2}+\left(\frac{m_{s}}{m_{b}}\right)^{2}\right]^{2}}
$$

All the quantities here are those measured at the GUT scale. Although this relation was found in our explicit $E_{6}$ GUT model, it actually does not depend on the details of the model. On the contrary, it turns out to have a much more generality (for example, [3, 4, 5]). It is a kind of an $S O(10)$ GUT relation similar to the famous $S U(5)$ relation $m_{b} / m_{\tau}=1$ [6], as the derivation shows which we give now.

This relation (11) results if the theory gives the following mass matrices for the up-quark, down-quark and charged-lepton sectors:

$$
\begin{aligned}
\bar{u}_{L} M_{u} u_{R} & =\mu_{u} \bar{u}_{L}\left(\begin{array}{cc}
O\left(\lambda^{4}\right) & f \lambda^{2} \\
O\left(\lambda^{2}\right) & 1
\end{array}\right) u_{R}, \\
\bar{d}_{L} M_{d} d_{R} & =\mu_{d} \bar{d}_{L}\left(\begin{array}{cc}
e \lambda^{2} & f \lambda^{2} \\
h & 1
\end{array}\right) d_{R}, \\
\bar{e}_{R} M_{e}^{\mathrm{T}} e_{L} & =\mu_{d} \bar{e}_{R}\left(\begin{array}{cc}
O\left(\lambda^{2}\right) & O\left(\lambda^{2}\right) \\
h & 1
\end{array}\right) e_{L},
\end{aligned}
$$

where we have written $2 \times 2$ matrices concentrating our attention only to the second and third generations, for simplicity, and we have normalized the 2-2 entries to be 1 by factoring out the mass scale parameters $\mu_{u}$ and $\mu_{d}$. $\lambda$ denotes a number of the order of the Cabibbo angle $\sin \theta_{\mathrm{C}} \sim 0.22$, and all the coefficients, $e, f, h$, are assumed to be of 
order $\lambda^{0}$. The point here is that (i) the second columns of $M_{u}$ and $M_{d}$ are commonly given by $\left(f \lambda^{2}, 1\right)^{\mathrm{T}}$, and (ii) the second rows of $M_{d}$ and $M_{e}^{\mathrm{T}}$ commonly by $(h, 1)$. The first property (i), being a proportionality condition only of the second columns of $M_{u}$ and $M_{d}$, will clearly hold if, as a sufficient condition, the third generation up and down quarks fall into a single representation of the GUT group $S O(10)$ or larger. The second property (ii) is satisfied if the right-handed down quark and the left-handed charged lepton in each generation are contained in a single $5^{*}$ of $S U(5)$. Indeed the equality of the 2-2 entries of $M_{d}$ and $M_{e}^{\mathrm{T}}$ is the same as the famous $m_{b}=m_{\tau}$ relation, and we are now demanding the same $S U(5)$ relation to hold also for the 2-1 entries, $h$.

Given these forms of the mass matrices in any case, we can find the following unitary matrices diagonalizing the matrices $M_{i} M_{i}^{\dagger}, U_{i}\left(M_{i} M_{i}^{\dagger}\right) U_{i}^{\dagger}=$ diagonal for $i=$ $u, d, e$ :

$$
\begin{aligned}
U_{u} & =\left(\begin{array}{cc}
1 & -f \lambda^{2} \\
f \lambda^{2} & 1
\end{array}\right), \\
U_{d} & =\left(\begin{array}{cc}
1 & -\frac{f+e h}{1+h^{2}} \lambda^{2} \\
\frac{f+e h}{1+h^{2}} \lambda^{2} & 1
\end{array}\right), \\
U_{e} & =\left(\begin{array}{cc}
\frac{1}{\sqrt{1+h^{2}}} & -\frac{h}{\sqrt{1+h^{2}}} \\
\frac{h}{\sqrt{1+h^{2}}} & \frac{1}{\sqrt{1+h^{2}}}
\end{array}\right),
\end{aligned}
$$

up to smaller corrections suppressed by a factor $\lambda^{2}$. Note that the down-quark matrix $M_{d}$ itself is diagonalized as

$$
\begin{aligned}
& U_{d} M_{d} U_{e}^{\dagger}=\left(\begin{array}{cc}
m_{s} & 0 \\
0 & m_{b}
\end{array}\right), \\
& m_{s}=\mu_{d} \frac{e-f h}{\sqrt{1+h^{2}}} \lambda^{2}, \quad m_{b}=\mu_{d} \sqrt{1+h^{2}}
\end{aligned}
$$

by using $U_{d}$ and the charged-lepton's $U_{e}$. This is due to the $S U(5)$ property (ii) mentioned above. Now, using Eqs. (4) and (5), we can calculate the element $V_{c b}$ of the CKM matrix $V=U_{u} U_{d}^{\dagger}$ and immediately find a relation

$$
V_{c b}=\frac{m_{s}}{m_{b}} h+O\left(\lambda^{4}\right) .
$$


This is almost the relation we desire. However, to reveal the $S O(10)$ property (i) working behind, let us re-derive this in another way.

For this purpose, we switch to the basis in which the up quark mass matrix is diagonalized, then the quark doublets are transformed as $\left(\bar{u}_{L}, \bar{d}_{L}\right) \rightarrow\left(\bar{u}_{L}, \bar{d}_{L}\right) U_{u}$ so that the down-quark mass matrix turns into

$$
M_{d}^{\prime}=U_{u} M_{d}=\left(\begin{array}{cc}
V_{c s} & V_{c b} \\
V_{t s} & V_{t b}
\end{array}\right)\left(\begin{array}{cc}
m_{s} & 0 \\
0 & m_{b}
\end{array}\right)\left(\begin{array}{cc}
U_{\mu 2} & U_{\mu 3} \\
U_{\tau 2} & U_{\tau 3}
\end{array}\right)
$$

where $V_{i j}$ are the matrix elements of the CKM matrix $V$, and $U_{\mu i}$ and $U_{\tau i}$ are those of the charged-lepton $U_{e}$. In this up-quark-diagonal basis, the 1-2 entry $M_{d 12}^{\prime}$ becomes zero up to $O\left(\lambda^{4}\right)$. This important fact comes from the $S O(10)$ property (i) in the above; indeed, the left multiplication of $U_{u}$ (together with the right multiplication of a suitable matrix) makes the up-quark mass matrix $M_{u}$ diagonal and so, taking also account of the $\lambda$-hierarchical structure of $M_{u}$, we see that the 1-2 entry of $U_{u} M_{u}$ vanishes up to an $O\left(\lambda^{4}\right)$ correction. But the $M_{u}$ and $M_{d}$ have the second columns exactly proportional to each other so that $U_{u} M_{d}$ should also have vanishing 1-2 entry up to $O\left(\lambda^{4}\right)$. Computing the 1-2 matrix element $M_{d 12}^{\prime}$ from the expression (7), we obtain

$$
V_{c s} m_{s} U_{\mu 3}+V_{c b} m_{b} U_{\tau 3}=0+O\left(\lambda^{4}\right) .
$$

or equivalently, using $V_{c s}=1+O\left(\lambda^{2}\right)$,

$$
-\frac{U_{\mu 3}}{U_{\tau 3}}=\frac{m_{b}}{m_{s}} V_{c b}+O\left(\lambda^{2}\right) .
$$

This is just the same relation as Eq. (6).

This Eq. (9) is nothing but the relation (1) announced at the beginning, provided that the MNS lepton mixing comes almost solely from the charged-lepton sector. The MNS matrix is generally given by $V_{\mathrm{MNS}}=U_{e} U_{\nu}^{\dagger}$ in terms of the above chargedlepton's $U_{e}$ and the neutrino's $U_{\nu}$ which diagonalizes the light neutrino Majorana mass matrix $M_{\nu}$ (as $U_{\nu}^{*} M_{\nu} U_{\nu}^{\dagger}=$ diagonal). So, the condition is that (iii) the neutrino diagonalization matrix $U_{\nu}$ is essentially a unit matrix:

$$
U_{\nu}=1+O\left(\lambda^{2}\right)
$$

This holds, for instance if the neutrino Majorana mass matrix $M_{\nu}$ takes the form of the hierarchical structure

$$
M_{\nu} \propto\left(\begin{array}{cc}
\lambda^{2 \alpha} & \lambda^{\alpha} \\
\lambda^{\alpha} & 1
\end{array}\right)
$$


in the fermion basis in which $M_{u}, M_{d}$ and $M_{e}$ take the forms (3). If the power $\alpha$ equals $2, M_{\nu}$ has the same structure as the up-quark mass matrix $M_{u}$ in Eq. (3) and the Eq. (10) results. Such a parallelism between $M_{\nu}$ and $M_{u}$ seems natural to appear in $S O(10)$ GUT models, although being not automatic from the symmetry alone, of course, since it depends on the structure of the right-handed neutrino Majorana mass matrix. A hierarchical structure (11) of $M_{\nu}$ comes out, for instance, in a supersymmetric $E_{6}$ GUT model studied by the present authors recently.

We have thus clarified how and under which conditions our relation (11) can be derived. It is actually remarkable that the relation (11), or the equivalent one (2), is in good agreement with the observed large lepton mixing angle $\theta_{\mu \tau}$ and the experimental data of quark masses and mixing. In Fig. 1, we show the value of $\sin ^{2} 2 \theta_{\mu \tau}$ which the relation (2) predicts for various values of the quark mass ratio $m_{s} / m_{b}$ and CKM matrix element $V_{c b}$ within their experimental errors.

We have now come to another important point. The relation (11) itself results from the conditions (i)-(iii) alone and is well satisfied by the experimental values. However, for the model satisfying these three conditions to really predict the large lepton mixing angle $\theta_{\mu \tau}$, or equivalently to reproduce the experimental value for the right-hand side (quark side) of the relation (1), it must realize the relation $h \simeq 1$. This is clear since the lepton mixing angle is given by $\tan \theta_{\mu \tau}=h$ as is seen from $U_{e}$ in Eq. (4).

The requirement $h \simeq 1$ gives a very non-trivial condition to the models. To see the point it is better to use explicit models realizing the above relations. We here consider two such models, 'generation flipped' $S O(10)$ model by Nomura and Yanagida (NY) [4], and ' $E$-twisted' $E_{6}$ model [3] by the present authors. As far as the second and third generations are concerned, these two models are essentially the same (although the latter model is more predictive, and superior at the point that it can additionally explain the largeness of $m_{t} / m_{b}$ ). In these models, the required hierarchical structure by powers of $\lambda$ of the mass matrices $M_{u}, M_{d}$ and $M_{e}$ in Eq. (3) can be realized by the Froggatt-Nielsen mechanism [0], and the powers of $\lambda$ are determined by the $U(1)$ quantum numbers assigned to the fermions. Thus the fermions belonging to the same multiplet must have the same $U(1)$ quantum numbers and hence the same powers of $\lambda$ in their masses. The mass terms at issue are

$$
\mu_{d} \bar{d}_{L 3}\left(h d_{R 2}+1 d_{R 3}\right)
$$


for down quarks, which should be compared with

$$
\mu_{u} \bar{u}_{L 3}\left(O\left(\lambda^{2}\right) u_{R 2}+1 u_{R 3}\right)
$$

for up quarks, where the numbers in the suffices denote the generation. The ratio $\lambda^{2}: 1$ between $u_{R 2}$ and $u_{R 3}$ for the latter is different from $h: 1$ between $d_{R 2}$ and $d_{R 3}$ which should be order 1 here. In the NY model the third generation fermions belong to a single multiplet 16 of the GUT group $S O(10)$ (the condition (i)). So, in order to have this different powers of $\lambda$ (the condition (ii)), the second generation up quark $u_{R 2}$ must belong to a different multiplet from that of down quark $d_{R 2}$. This is actually the case in the NY model, where the up-quark $u_{R 2} \in S U(5) \mathbf{1 0}$ belong to a 16 as usual while the down-quark $d_{R 2} \in S U(5) 5^{*}$ come from another multiplet 10 of $S O(10)$. It is this mechanism of violating the parallel generation structure that is required for explaining the observed large lepton mixing.

It is interesting to see how the same is realized in the $E_{6}$ model of ours. It is always possible to find an $S O(10)$ group under which a $\mathbf{1 0}$ and a $\mathbf{5}^{*}$ of $S U(5)$ are combined into a 16. Therefore, although in the NY model the third generation fermions are in a 16 while the second generation 10 and $5^{*}$ of $S U(5)$ come separately from 16 and 10 of $S O(10)$, the converse is also true; namely, if we take another $S O(10)$ group, which may be called 'flipped $S O(10)$ ', then the second generation fermions fall into a single $\mathbf{1 6}$ while the third generation fermions are separated into $\mathbf{1 6}$ and 10. (In the case of $E_{6}$, moreover, the two $S O(10)$ groups, the original one and the flipped one, are mutually converted into each other by an $S U(2)_{E}$ rotation with angle $\pi$ contained in $E_{6}$, hence explaining the name ' $E$-twisting'.) This is exactly what happens in our $E_{6}$ model for the $S O(10)$ subgroup, which is chosen by the first step spontaneous breaking $E_{6} \rightarrow S O(10)$; among three generations, each belonging to a $\mathbf{2 7}$ of $E_{6}$ in the beginning, the second and first generation fermions each fall into a $\mathbf{1 6}$ under the $S O(10)$ subgroup after the breaking, while the third generation splits into $S U(5) \mathbf{1 0} \subset \mathbf{1 6}$ and $S U(5) \mathbf{5}^{*} \subset \mathbf{1 0}$.

We suspect that the observed very large mixing in the lepton/neutrino sector indicates the existence of such a 'flipping' or 'twisting' structure as an essential ingredient of the GUT models. Remarkably, the $E_{6}$ model already prepares such a mechanism intrinsically; the fundamental representation $\mathbf{2 7}$ contains $\mathbf{1 6}$ and $\mathbf{1 0}$ of $S O(10)$ and so two $S U(5) 5^{*}$ s. They can be mixed or even twisted completely by the internal rotation in $E_{6}$. 
Finally we add a comment that another relation can be obtained from the same conditions if we include the first generation also. Then, the mass matrices become $3 \times 3$, and the previous condition (i) is generalized to the proportionality of the third column of $M_{u}$ and $M_{d}$ while the second property (ii) for $M_{d}$ and $M_{e}$ and (iii) are kept the same. Then the same argument as before leads to a relation

$$
\tan \theta_{\mu \tau}=\frac{V_{u b}}{V_{u s}} \frac{m_{b}}{m_{s}}
$$

This also roughly agrees with the experimental data, although the inclusion of the first generation data becomes less reliable generally.

To conclude, we have found in this letter the novel relations between the quark and lepton mixing angles in GUTs. The relations hold under the simple conditions, especially, if the third generation up and down quarks come from a single multiplet. It clearly requires $S O(10)$ or larger unified gauge groups. We have also shown that the conditions are naturally realized with the 'twisted' generation structure of the second and third generations. The experimental agreement of the presented relations may corroborate these interesting GUT structures.

\section{Acknowledgments}

We would like to thank to Y. Nomura, N. Okamura and T. Yanagida for stimulating discussions. We also thank the Summer Institute 99 held at Yamanashi, Japan. M. B., T. K. and K. Y. are supported in part by the Grants-in-Aid for Scientific Research No. 09640375, No. 10640261, and the Grant-in-Aid No. 9161, respectively, from the Ministry of Education, Science, Sports and Culture, Japan.

\section{References}

[1] The Super-Kamiokande Collaboration, Y. Fukuda et al, Phys. Rev. Lett. 81 (1998) 1562.

[2] For example, K.S. Babu and R.N. Mohapatra, Phys. Rev. Lett. 70 (1993) 2845; H. Fritzsch and Z.Z. Xing, Phys. Lett. 372B (1996) 265; M. Fukugita, M. Tanimoto and T. Yanagida, Phys. Rev. D57 (1998) 4429; M. Bando, T. Kugo and 
K. Yoshioka, Phys. Rev. Lett. 80 (1998) 3004; J. Sato and T. Yanagida, Phys. Lett. 430B (1998) 127; Q.Y. Liu and A.Yu. Smirnov, Nucl. Phys. B524 (1998) 505; V. Barger, T.J. Weiler and K. Whisnant, Phys. Lett. 427B (1998) 97; G. Altarelli and F. Feruglio, JHEP 9811 (1998) 021; T. Blazek, S. Raby and K. Tobe, Phys. Rev. D60 (1999) 113001; L. Hall, H. Murayama and N. Weiner, hep-ph/9911341.

[3] M. Bando and T. Kugo, Prog. Theor. Phys. 101 (1999) 1313; M. Bando, T. Kugo and K. Yoshioka, hep-ph/0003220.

[4] Y. Nomura and T. Yanagida, Phys. Rev. D59 (1998) 017303.

[5] C.H. Albright, K.S. Babu and S.M. Barr, Phys. Rev. Lett. 81 (1998) 1167; C.H. Albright and S.M. Barr, Phys. Lett. 452B (1999) 287.

[6] M.S. Chanowitz, J. Ellis and M.K. Gaillard, Nucl. Phys. B128 (1977) 506; H. Arason et al., Phys. Rev. Lett. 67 (1991) 2933; V. Barger, M.S. Berger and P. Ohmann, Phys. Rev. D47 (1993) 1093; M. Carena, S. Pokorski and C.E.M. Wagner, Nucl. Phys. B406 (1993) 59.

[7] C.D. Froggatt and H.B. Nielsen, Nucl. Phys. B147 (1979) 277. 


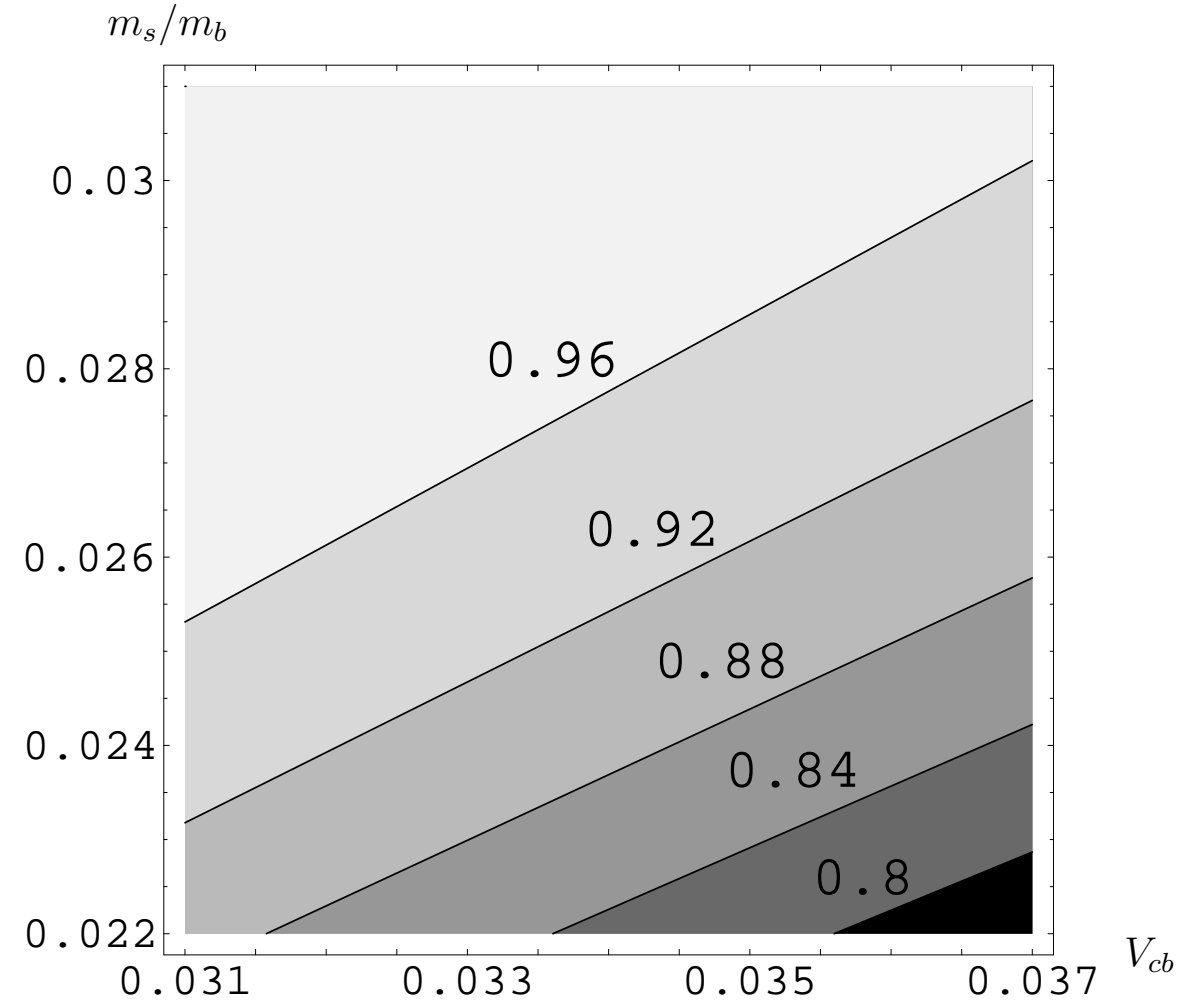

Figure 1: The prediction of the lepton 2-3 mixing angle $\sin ^{2} 2 \theta_{\mu \tau}$ from the relation (2). The square parameter region is the experimental uncertainties of $m_{s} / m_{b}$ and $V_{c b}$. In almost all range, the relation is consistent with the observations. 